\title{
Adenoidectomy: Conventional or Endoscopic Assisted?
}

\section{Anuj K Goel}

\begin{abstract}
Objectives: Adenoid hypertrophy is known as the most common cause of nasal obstruction in children; thus, adenoidectomy is one of the most commonly performed surgical procedures in the pediatric population. We performed both conventional and endoscopic-assisted adenoidectomies in 54 patients. Our objectives were to compare the efficacy of both methods in terms of subjective and objective relief of symptoms, safety, recurrence, and postoperative morbidity.
\end{abstract}

Materials and methods: Fifty-four patients were studied and were diagnosed depending upon clinical examination and radiological investigation. The patients were operated by either conventional or endoscopic-assisted adenoidectomy (EAA). Intraoperative and postoperative complications were looked for. After discharge from the hospital, the patients were called for follow-up on 1st week, 3rd week, 2nd and 4th month, and patients were asked for relief of symptoms and examined for nasal disease and for recurrence.

Results: Adenoid hypertrophy was seen commonly in children and the most common presenting complaints were nasal obstruction, mouth breathing, and snoring. The patients were diagnosed to have chronic adenoiditis, chronic adenotonsillitis, with or without chronic suppurative otitis media. More number of patients became symptom free with EAA as compared with conventional adenoidectomy.

Conclusion: Conventional adenoidectomy and EAA both have comparable success rates. Endoscopic-assisted adenoidectomy allows complete removal of adenoid tissue, thereby reducing the chances of developing recurrent adenoid obstructive symptoms and reducing the bacterial reservoir in the nasopharynx.

Keywords: Adenoid, Nasal obstruction, Nasopharynx.

How to cite this article: Goel AK. Adenoidectomy: Conventional or Endoscopic Assisted? Clin Rhinol An Int J 2017;10(2):74-77.

Source of support: Nil

Conflict of interest: None

\section{INTRODUCTION}

Adenoidectomy is among the most common operations performed in children worldwide. In addition to

\section{Associate Professor}

Department of ENT, Saraswathi Institute of Medical Sciences Hapur, Uttar Pradesh, India

Corresponding Author: Anuj K Goel, Associate Professor Department of ENT, Saraswathi Institute of Medical Sciences Hapur, Uttar Pradesh, India, Phone: +9109897673888, e-mail: dranujgoel12@gmail.com this surgical work load, physician consultations for the associated symptoms of nasal obstruction, snoring, and nasal discharge account for significant number of visit to otolaryngology and allergic specialists. These symptoms can impair a child's quality of life and may have unfavorable developmental effects that predispose the child to sleep-related breathing abnormalities later on. Airway obstruction related to adenotonsillar hypertrophy can be associated with long-term consequences, such as failure to thrive and sleep disturbance leading to inability to concentrate, day-time somnolence, and low results of psychometric tests. ${ }^{1}$

Adenoid hypertrophy is believed to play a dual role in the etiology of otitis media (OM). First, it may serve as mechanical obstruction to the orifice of the Eustachian tube (ET) in the naospharynx, thereby limiting the drainage of the middle ear. Second, adenoids may serve as a reservoir of infection, providing a bacterial source for OM. Adenoidectomy, as adjuvant surgical therapy, may improve the outcome of OM by either removing this bacterial focus or by improving the function of the ET. ${ }^{2}$

Adenoidectomy in children is a difficult operation to perform well. Conventional adenoidectomy is commonly performed by blind digital palpation of the adenoid mass in the nasopharynx and then removal by using adenoid curettes with hemostasis by way of postnasal packing. Complete removal is difficult to determine due to blind nature of conventional method. ${ }^{3}$ Endoscopic-assisted adenoidectomy (EAA) is a natural progression of this technology to allow a more complete adenoidectomy; thus, EAA technique is advocated for use as an adjunct to a more complete adenoidectomy.

\section{MATERIALS AND METHODS}

The present prospective study between EAA and conventional adenoidectomy was conducted in 54 patients with adenoid hypertrophy diagnosed by clinical examination, confirmed by X-ray soft tissue nasopharynx lateral view. The cases of adenoid hypertrophy diagnosed clinically as well as radiologically were included in the study. Conditions that mimic adenoid hypertrophy clinically like angiofibroma, deviated nasal septum, allergic rhinitis, and hypertrophic turbinates and patients with bleeding disorders were excluded from the study. Detailed history and clinical examination was done in selected cases. Routine blood investigations like hemoglobin \%, 
total leukocyte count, differential percentage leukocyte count, erythrocyte sedimentation rate, bleeding time, computed tomography, urine for albumin, sugar, and microscopy, human immunodeficiency virus, surface antigen of the hepatitis $B$ virus, and other investigations like X-ray nasopharynx lateral view and chest $\mathrm{X}$-ray as and when required were done. Cases with acute infections were treated on medical line and then they were taken up for surgery. Written and informed consent was taken prior to surgery. The cases were grouped randomly into group I for conventional surgery and group II for EAA, with 27 cases each. In conventional adenoidectomy, general anesthesia was employed with orotracheal tube. Patient was placed supine on the operating table. BoyleDavis' mouth gag with tongue blade was applied in the oral cavity which was supported by Daraffin's bipods. A more neutral position of the neck was given. The adenoids were palpated with an index finger. A St. Clair Thomson adenoid curette with guard was then inserted into the nasopharynx, gently positioned against the posterior border of the nasal septum, and swept downward. As the main mass of adenoids was curetted, the blade of the curette was brought forward to avoid running it down the posterior pharyngeal wall and stripping off the mucosa. The nasopharynx was palpated and any adenoid remnants if found were curetted. Then, a pack was placed in the nasopharynx. After achieving hemostasis, pack in the nasopharynx was removed. Patient was extubated after confirming that there was absolutely no bleeding from nasopharynx. In EAA, nasal cavity was packed with $2 \%$ xylocaine with adrenaline-rinsed cotton pledgets. The main bulk of the adenoid tissue was removed by curettage as in group I. Then the patient's position was changed from Rose's position to head end elevation position. The nasal packs (cotton pledgets) were removed. Subsequently, a 4 or $2.7 \mathrm{~mm} 0^{\circ}$ endoscope was inserted intranasally, tissue at the superior portion of the nasopharynx and at the opening of the ET was removed piece by piece using a straight or $45^{\circ}$ Blakesley forceps under direct visualization. Hemostasis was evaluated and achieved via cauterization under direct endoscopic visualization. After achieving complete hemostasis, patient was extubated. Depending upon the type of surgery, intraoperative complications were looked for. All patients received a course of antibiotics postoperatively for 5 days. Postoperative complications were recorded. The patients were discharged when found to be fit and were strictly called for follow-up on 1st week, 3 rd week, 2nd, and 4th month from the date of surgery. On the following visits, the patients were asked for relief of symptoms and examined for cold spatula test, nasal discharge, and recurrence. The observations made from either surgery were compared.

\section{RESULTS}

The present study included 54 cases, 27 each in two groups, the first group of patients had undergone conventional adenoidectomy and the second group had undergone EAA. The patients' age ranged from 3 to 14 years. The mean age of group I was 8.3 years and that of group II was 9.5 years. The combined mean was 8.9 years. Highest number of patients belong to the age group 5 to 10 years (31 in number) followed by those in age group 10 to 15 years (17 in number) followed by those in age group $<5$ years (6 in number). In the present study, the youngest patient was 3 years old and the oldest was 14 years old. There were 24 males accounting for $44.4 \%$ totally and 30 females accounting for 55.5\% totally. In group I, 33.3\% were males, $66.6 \%$ were females, while in group II, $55.5 \%$ were males and $44.4 \%$ were females. Nasal obstruction was the single most common complaint in the patients of both groups affecting all the 54 patients; 42 patients had nasal discharge, 19 patients had associated throat pain, 10 patients had associated ear discharge, but only 4 patients had decreased hearing. During the 1st week follow-up, only three patients (11.1\%) in group II had no symptoms, whereas no patient was free of symptom in group I. In 3rd week follow-up, 8 (29.6\%) patients in group I were symptom free and 15 (55.5\%) patients were symptom free in group II. By 2 months, 20 patients (74.1\%) were free of symptoms in group II, whereas only 16 (59.2\%) were in group I. At the end of 4 months, 20 patients (74.1\%) in group I and 26 patients (96.3\%) in group II were symptoms free. So at 4 months follow-up, 20 patients (74.1\%) in group I were symptom free as compared with 26 patients (96.3\%) in group II, having p-value 0.04 , which is significant.

\section{DISCUSSION}

Adenoidectomy is one of the most common procedures performed by otolaryngologists. It has been performed for more than 100 years. ${ }^{5}$ For many years, it was performed by strictly a transoral approach with curettes, an adenoid punch, and an adenotome. One advantage of performing a more complete adenoidectomy is decreasing the bacterial reservoir, which affects children with OM, nasopharyngitis, and possibly sinusitis as well. It would also be beneficial in preventing recurrent airway obstructive symptoms. ${ }^{4}$ Adenoidectomy done using adenotome or adenoid curette to remove hypertrophied adenoid tissue does not always produce complete removal of the adenoid, especially the superior and peritubal portions of adenoid that obstruct the nasopharynx and the orifice of the ET. Overly aggressive curettage may result in inadvertent damage to muscle, the choana, or other structures. Other modifications of this technique have been reported. 
Pearl and Manoukian ${ }^{6}$ identified the presence of choanal adenoids in $9.4 \%$ of the adenoidectomy population and suggested this tissue is missed using traditional "blind" adenoidectomy techniques. Conversely, if an attempt is made to remove the tissue with too much force, it is possible to injure the posterior choana and the muscular layer of the posterior pharyngeal wall. This may result in massive hemorrhage. If the ET is damaged, the dysfunction can be irreversible. Drake et $\mathrm{al}^{7}$ found that a curved uterine curette was useful to remove peritubal residual adenoid tissue and avoid injury to the ET. They concluded that the technique could remove adenoid tissue laterally in the nasopharynx and inferior to the Rosenmuller's fossa. However, this technique was also performed in a blind manner and thus the possibility of complications still existed. Furthermore, steps to stop nasopharyngeal hemorrhage were also blind. To obtain better visualization of the adenoids, Pearl and Manoukian ${ }^{6}$ reported the adjuvant use of an indirect laryngeal mirror to light the dark nasopharyngeal space. However, the mirror image was no better than the direct visualization. Additionally, instruments used in the operation could affect the stability of the mirror image. Becker et $\mathrm{al}^{8}$ reported using endoscopic adenoidectomy for serous OM in young adolescents and adults. The enlarged adenoids were removed piece by piece with a Blakesley forceps under transnasal endoscopic visualization. This caused increased blood loss, prolonged time needed for anesthesia, and increased postoperative complications. Nayak et $\mathrm{al}^{9}$ adopted transnasal adenoidectomy in a patient with Scheie's syndrome. In our study, 54 cases were operated by either conventional method or endoscopic-assisted technique. The cases were grouped into group I for conventional surgery and group II for EAA. In our study of 54 cases, mean age was found to be 8.9 years, which compares well with the study by Georgalas et $\mathrm{al}^{1}$ where the mean age of the patients was 8.4 years. In our study, a female preponderance was seen with $55.5 \%$ of females and $44.4 \%$ of males, which compares well with the study of Flanary ${ }^{10}$ in which females were $51.6 \%$ and males were $43.3 \%$. We observed nasal obstruction, mouth breathing, snoring, followed by nasal discharge as commonest symptoms whereas Georgalas et $\mathrm{al}^{1}$ reported mouth breathing, snoring, rhinorrhea, and cough. Huang et $\mathrm{al}^{11}$ reported in their study nasal obstruction, mouth breathing, and snoring, as was also observed by us. Mitchell et $\mathrm{al}^{12}$ in their study reported mean hospital stay of 1.4 days after adenoidectomy. In our study, the average hospital stay was 1.3 days for conventional adenoidectomy and 1.16 days for EAA, correlating with previous reports. In our study, two patients had recurrence of bilateral ear discharge and three patients had recurrence of nasal obstruction and nasal discharge after 6 months of conventional adenoidectomy, while in EAA there was no such case of recurrence which correlates with the study by Cannon et al, ${ }^{4}$ which states that complete adenoidectomy involves decreasing the bacterial reservoir, which affects the children with OM, nasopharyngitis, and possibly sinusitis as well. In our study, about $55 \%$ of patients became symptom free by the end of 3 weeks who underwent EAA as compared with conventional method where only $29.6 \%$ became symptom free. By the end of 4 months, $74.1 \%$ of patients became free of symptoms in conventional surgery, but $96.3 \%$ of patients who underwent EAA became symptom free, which correlates with the study by Becker et $\mathrm{al}^{8}$ in which $92 \%$ cases were free of OM after endoscopic adenoidectomy. The use of only endoscopic equipments allows the adenoid to be removed piece by piece. However, in patients with a very large adenoid, endoscopic removal requires more time than conventional surgery, which prolongs the need for anesthesia and increases its risk, as studied by Huang et $\mathrm{al}^{11,13}$ The combination of conventional and endoscopic approaches in these patients will shorten the operative time to remove the adenoid. Shin and Hartnick ${ }^{14}$ studied three cases in which operative time for the adenoidectomy portion of the procedure, including endoscopic equipment setup and photodocumentation, was 10 to 15 minutes. In our study also, there is only a minimal increase in the operating time taken for EAA. Cannon et $\mathrm{al}^{4}$ found that after conventional adenoidectomy, there is always residual tissue in the posterior superior choana of the nose and nasopharynx. Conventional adenoidectomy combined with endoscopic-assisted removal allows complete removal of adenoid tissue without a significant increase in the operative time, blood loss, or association with any postoperative complications. In our study, these observations correlate with the previous study.

\section{CONCLUSION}

Conventional adenoidectomy in experienced hands and EAA have comparable success rates, but conventional adenoidectomy followed by endoscopic-assisted removal of residual adenoid tissue achieves complete removal and hemostasis under direct vision. It also prevents injury to posterior pharyngeal wall and ET orifice so EAA can be used as an adjunct to perform a more complete adenoidectomy.

\section{REFERENCES}

1. Georgalas C, Thomas K, Owens C, Abramovich S, Lack G. Medical treatment forrhinosinusitis associated with adenoidal hypertrophy in children: an evaluation of clinical response and changes on magnetic resonance imaging. Ann Otol Rhinol Laryngol 2005 Aug;114(8):638-644. 
2. Nguyen LHP, Manoukian JJ, Yoskovitch A, Al-Sebeih KH. Adenoidectomy - selection criteria for surgical cases of otitis media. Laryngoscope 2004 May;114(5):863-866.

3. Walker P. Pediatric adenoidectomy under vision using suction diathermy ablation. Laryngoscope 2001;111(12)2173-2177.

4. Cannon CR, Replogle WH, Schenk MP. Endoscopicassisted adenoidectomy. Otolaryngol Head Neck Surg 1999 Dec;121(6):740-744.

5. Curtin JM. The history of tonsil and adenoid surgery. Otolaryngol Clin North Am 1987 May:20(2):415-419.

6. Pearl AJ, Manoukian JJ. Adenoidectomy: indirect visualization of choanal adenoids. J Otol Laryngol 1994 Jun;23(3): 221-224.

7. Drake AF, Fishcer ND, Newton D. Peritubal adenoidectomy. Laryngoscope 1993 Nov;103(11 Pt 1):1291-1292.

8. Becker SP, Roberts N, Collgianese D. Endoscopic adenoidectomy for relief of serous otitis media. Laryngoscope 1992 Dec;102(12 Pt 1):1379-1384.
9. Nayak DR, Balakrishnan R, Adolph S. Endoscopic adenoidectomy in a case of Scheie syndrome. Int J Pediatr Otrohinolaryngol 1998 Jul;44(2):177-181.

10. Flanary VA. Long-term effect of adenotonisllectomy on quality of life in paediatric patients. Laryngoscope 2003 Oct;113(10):1639-1644.

11. Huang HM, Chao MC, Chen YL, Hsiao HR. A combined method of conventional and endoscopic adenoidectomy. Laryngoscope 1998 Jul;108(7):1104-1106.

12. Mitchell RB, Pereira KD, Friedman NR, Lazar RH. Outpatient adenotonsillectomy - is it safe in children younger than 3 years? Arch Otolaryngol Head Neck Surg 1997 Jul;123(7): 681-683.

13. Chen CW, Lee FP, Chen HT, Huang HM. A combined method of transoral and transnasal endoscopic adenoidectomy. Tzu Chi Med J 2002 Oct;14(5):289-294.

14. Shin JJ, Hartnick CJ. Pediatric endoscopic transnasal adenoid ablation. Ann Otol Rhinol Laryngol 2003 Jun;112(6):511-514. 